\title{
New-onset seizure during and after brain tumor excision: a risk assessment analysis
}

\author{
Soliman Oushy, BS, ${ }^{1}$ Stefan H. Sillau, PhD, ${ }^{1}$ Douglas E. Ney, MD, ${ }^{2}$ Denise M. Damek, MD, ${ }^{2}$ \\ A. Samy Youssef, MD, PhD, ${ }^{1}$ Kevin O. Lillehei, MD, ${ }^{1}$ and D. Ryan Ormond, MD ${ }^{1}$ \\ Departments of ${ }^{1}$ Neurosurgery and ${ }^{2}$ Neurology, University of Colorado School of Medicine, Aurora, Colorado
}

\begin{abstract}
OBJECTIVE Prophylactic use of antiepileptic drugs (AEDs) in seizure-naïve brain tumor patients remains a topic of debate. This study aimed to characterize a subset of patients at highest risk for new-onset perioperative seizures (i.e., intraoperative and postoperative seizures occurring within 30 days of surgery) who may benefit from prophylactic AEDs.

METHODS The authors conducted a retrospective case-control study of all adults who had undergone tumor resection or biopsy at the authors' institution between January 1,2004, and June 31,2015. All patients with a history of preoperative seizures, posterior fossa tumors, pituitary tumors, and parasellar tumors were excluded. A control group was matched to the seizure patients according to age ( \pm 0 years). Demographic data, clinical status, operative data, and postoperative course data were collected and analyzed.
\end{abstract}

RESULTS Among 1693 patients who underwent tumor resection or biopsy, 549 (32.4\%) had never had a preoperative seizure. Of these 549 patients, $25(4.6 \%)$ suffered a perioperative seizure (Group 1). A total of 524 patients $(95.4 \%)$ who remained seizure free were matched to Group 1 according to age ( \pm 0 years), resulting in 132 control patients (Group 2), at an approximate ratio of 1:5. There were no differences between the patient groups in terms of age, sex, race, relationship status, and neurological deficits on presentation. Histological subtype (infiltrating glioma vs meningioma vs other, $p$ $=0.041)$, intradural tumor location $(p<0.001)$, intraoperative cortical stimulation $(p=0.004)$, and extent of resection (less than gross total, $p=0.002$ ) were associated with the occurrence of perioperative seizures.

CONCLUSIONS While most seizure-naïve brain tumor patients do not benefit from perioperative seizure prophylaxis, such treatment should be considered in high-risk patients with supratentorial intradural tumors, in patients undergoing intraoperative cortical stimulation, and in patients in whom subtotal resection is likely.

https://thejns.org/doi/abs/10.3171/2017.2.JNS162315

KEY WORDS seizure; prophylaxis; tumor; brain; epilepsy; oncology

$\mathrm{S}$ EIZURES occur in $35 \%-70 \%$ of brain tumor patients regardless of tumor type and location, while they only occur in $4 \%$ of the general population..$^{15,23}$ Of those brain tumor patients who do not initially present with a seizure, $6 \%-45 \%$ eventually experience seizures. ${ }^{5}$ Interestingly, preoperative seizures have been shown to be a favorable prognostic survival factor, possibly because they promote early recognition and treatment. ${ }^{20,22}$ On the other hand, perioperative seizures, that is, those occurring in patients within 30 days of surgery, add significant morbidity. ${ }^{12,16,26}$ While there is little controversy regarding antiepileptic drug (AED) use in patients presenting with seizures, the prophylactic use of AEDs in the management of brain tumor patients with no history of seizures remains a topic of debate.

The American Academy of Neurology (AAN) has, for a number of years, recommended AED use only in tumor patients with a documented seizure history, ${ }^{12}$ but given the morbidity associated with perioperative seizures, many neurosurgeons continue to use AEDs in the perioperative setting. In fact, as many as $81 \%$ of neurosurgeons have reported prescribing prophylactic AEDs to patients without a history of seizures..$^{13}$ Some studies have shown AEDs to be ineffective in preventing perioperative seizures in patients with brain tumors. ${ }^{10,11,13,31,37}$ In other studies, however, AED use has been shown to effectively prevent such

ABBREVIATIONS AAN = American Academy of Neurology; AED = antiepileptic drug; GTR = gross-total resection; STR = subtotal resection.

SUBMITTED September 13, 2016. ACCEPTED February 15, 2017.

INCLUDE WHEN CITING Published online July 28, 2017; DOI: 10.3171/2017.2.JNS162315. 


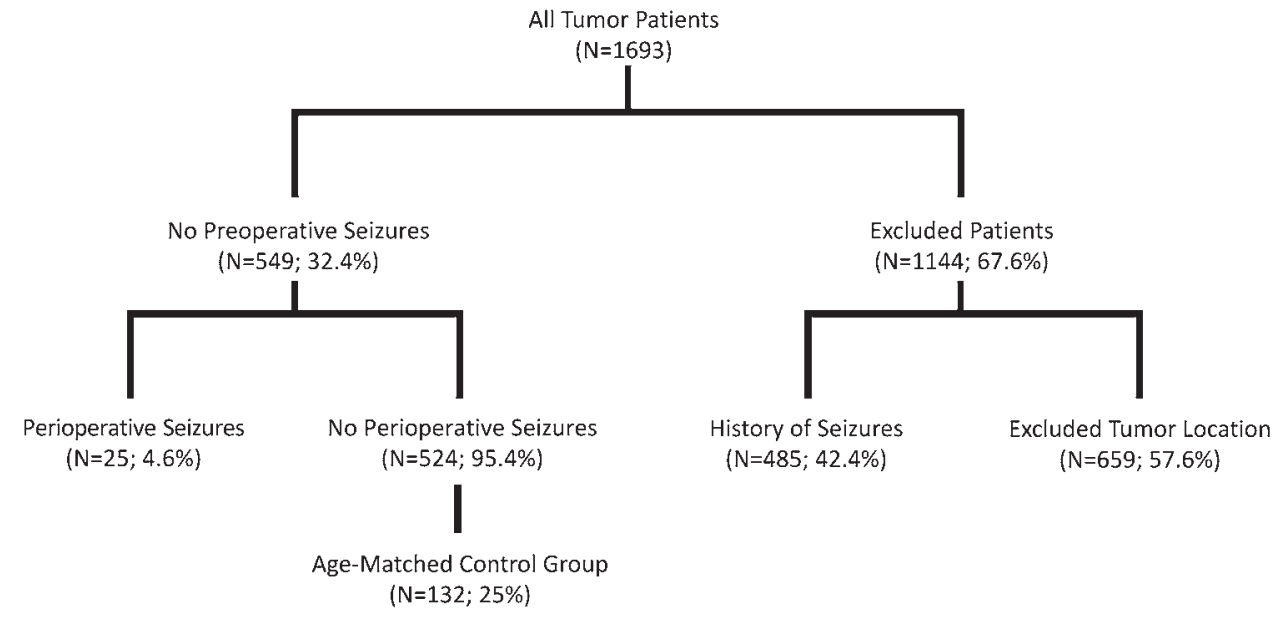

FIG. 1. Algorithm for patient selection.

seizures. ${ }^{14,28,34,35,39}$ This contrasting evidence suggests that if there is a benefit to using prophylactic AEDs in tumor patients without a history of seizures before surgery, their use is probably beneficial in only a subset of patients. Unfortunately, only limited evidence is available to determine which patients should be treated.

The aim of this study was to identify and characterize a subset of patients at highest risk for new-onset perioperative seizures who may benefit from prophylactic AEDs.

\section{Methods}

\section{Description of Patient Population}

Institutional review board approval was obtained per the guidelines of the University of Colorado School of Medicine. For the period from January 1, 2004, to June 31,2015 , we searched the electronic medical record for all adults with brain tumors who had undergone excision or biopsy at the University of Colorado Hospital. Patient charts were retrospectively reviewed. All patients with a history of seizures prior to tumor excision or biopsy were excluded from the study. In addition, patients with posterior fossa tumors, pituitary tumors, and parasellar tumors were excluded.

Seizure-naïve patients were divided into 2 groups based on their perioperative seizure status. "Perioperative seizure" was defined as a seizure within 30 days of surgery and included intraoperative and postoperative events. Group 1 comprised patients with perioperative seizures, whereas Group 2 comprised patients with no perioperative seizures. "Perioperative seizures" were defined by clinical manifestations including generalized involuntary movements, altered mental status, or abnormal motor or sensory phenomena. If a seizure was suspected but clinical manifestations were inconclusive, an abnormal ("positive") electroencephalograph was used to confirm the diagnosis. "Intraoperative seizures" refer to seizures recorded on electroencephalography during intraoperative cortical mapping procedures. "Postoperative seizures" were all other seizures during the 30-day postoperative period. This study was designed as a casecontrol study, with each patient with a perioperative sei- zure event age matched with all patients in the control arm (Fig. 1).

\section{Outcomes}

We compared the 2 patient groups to identify potential differing characteristics. The characteristics compared included age, histological diagnosis, tumor location, medications, extent of resection, and use of intraoperative stimulation. All patients had available pre- and postoperative CT and MRI studies to determine tumor location and extent of resection. Tumor histology was obtained from final pathology reports in the medical record. With regard to the type of surgical intervention, patients were categorized as having undergone gross-total resection (GTR), subtotal resection (STR), or biopsy, as reported by the senior surgeon in the operative report in the electronic medical record.

Given the small sample size, we elected to modify several categorical variables by combining 2 or more categories including race, relationship status, histological subtype, and extent of resection. Race was classified as white or nonwhite. Relationship status was classified as married or other. Histological diagnoses were classified as infiltrating glioma, meningioma, or other. Infiltrating gliomas included WHO Grade II or higher tumors since our patient cohort lacked any Grade I gliomas. The histological category "other" included metastases and nonglial primary central nervous system tumors. Patients were divided into 2 different treatment groups: GTR or all other surgical interventions (that is, STR or biopsy).

\section{Statistical Methods}

Since the study was case-control, exposure variables were compared between seizure and nonseizure groups. For binary exposure variables, odds ratios could be used to estimate the effect of exposure on seizure. Most exposure variables were categorical, so 2-way frequency tables, with percentages for exposure conditional on seizure status, were presented. Association between seizure and exposure was tested with a chi-square or Fisher's exact association test, as necessary. Odds ratios and their $95 \%$ 
TABLE 1. Summary of population characteristics

\begin{tabular}{lccc}
\hline \multicolumn{1}{c}{ Characteristic } & Group 1 & Group 2 & p Value \\
\hline No. of patients & 25 & 132 & \\
\hline Median age in yrs (range) & $54(15-77)$ & $56(14-77)$ & 0.4660 \\
\hline Sex (\% male) & $60.0 \%$ & $56.1 \%$ & 0.7155 \\
\hline Race (\% white) & $88.0 \%$ & $88.5 \%$ & 1.0000 \\
\hline Relationship status (\% married) & $56.5 \%$ & $72.5 \%$ & 0.1357 \\
\hline \% patients w/ focal motor, & $92.0 \%$ & $84.1 \%$ & 0.5359 \\
$\quad$ sensory, or speech deficit & & & \\
\hline Site of tumor & & & \\
\hline Rt hemisphere & $36.0 \%$ & $45.5 \%$ & 0.3825 \\
\hline Lt hemisphere & $60.0 \%$ & $45.5 \%$ & 0.1819 \\
\hline Frontal & $64.0 \%$ & $47.0 \%$ & 0.1184 \\
\hline Temporal & $32.0 \%$ & $25.8 \%$ & 0.5179 \\
\hline Parietal & $20.0 \%$ & $22.0 \%$ & 0.8265 \\
\hline Occipital & $4.0 \%$ & $12.9 \%$ & 0.3102 \\
\hline Intradural & $84.0 \%$ & $41.7 \%$ & 0.0001 \\
\hline Extradural & $20.0 \%$ & $45.5 \%$ & 0.0178 \\
\hline Seizure prophylaxis (any agent) & $95.7 \%$ & $83.0 \%$ & 0.2026 \\
\hline Intraop cortical stimulation & $24.0 \%$ & $9.2 \%$ & 0.0445 \\
\hline Extent of resection & & & \\
\hline GTR & $32.0 \%$ & $64.9 \%$ & 0.0021 \\
\hline STR or biopsy & $68.0 \%$ & $35.1 \%$ & 0.0021 \\
\hline$\quad$ & &
\end{tabular}

confidence intervals were calculated for binary exposure variables; exact methods were used because of some small sample sizes. Means and t-tests were used to compare age between seizure and nonseizure groups. Within the seizure group, frequencies for type and timing of seizure and intraoperative cortical stimulation were presented, and the association between timing of seizure and intraoperative cortical stimulation was tested. Because of the relatively small sample sizes, $\mathrm{p}$ values less than 0.05 were considered significant and p values between 0.05 and 0.10 were considered marginally statistically nonsignificant.

\section{Results \\ Population}

Of 1693 patients who had undergone tumor resection or biopsy, 659 (38.9\%) were excluded based on tumor location. A total of 549 patients were identified as seizure naïve prior to surgery. Among those patients, we identified $25(4.6 \%)$ who had suffered a perioperative seizure (Group 1), leaving 524 patients $(95.4 \%)$ who had never suffered a seizure event to serve as controls. The control group was matched to Group 1 according to age ( \pm 0 years), resulting in 132 control patients (Group 2) at an approximate ratio of 1:5. A comparison of characteristics between the 2 patient groups is shown in Table 1. There were no differences between patient groups in terms of age, sex, race, relationship status, and neurological deficits on presentation.

A majority $(84.8 \%)$ of all patients received perioperative prophylactic AEDs. The most frequently used AED was levetiracetam. Initiation of seizure prophylaxis was
TABLE 2. Predictors of perioperative seizures

\begin{tabular}{lllc}
\hline \multicolumn{1}{c}{ Variable } & OR & \multicolumn{1}{c}{$95 \% \mathrm{Cl}$} & $\mathrm{p} \mathrm{Value}$ \\
\hline Tumor histology & & & 0.04 \\
\hline \multicolumn{1}{c}{ Infiltrating glioma vs meningioma } & 3.57 & $1.08-15.24$ & 0.031 \\
\hline Infiltrating glioma vs other & 0.882 & $0.19-5.51$ & 1.00 \\
\hline Intradural tumor location & 7.35 & $2.28-30.74$ & $<0.001$ \\
\hline Extradural tumor location & 0.300 & $0.084-0.895$ & 0.018 \\
\hline STR or biopsy vs GTR & 3.93 & $1.46-11.26$ & 0.002 \\
\hline Intraop cortical stimulation & 3.13 & $1.04-9.34$ & 0.004 \\
\hline
\end{tabular}

not significantly associated with perioperative seizures ( $\mathrm{p}$ $=0.2026$ ). Seizure prophylaxis was administered in $95.7 \%$ of Group 1 patients compared with only $82.9 \%$ of Group 2 patients. In Group 1, 44.0\% of patients were on phenytoin prophylaxis compared with $15.9 \%$ in Group $2(\mathrm{p}=0.0014)$. Of the Group 1 patients on phenytoin, $54.5 \%$ were noted to have subtherapeutic phenytoin serum levels compared with $15.4 \%$ of the Group 2 patients $(\mathrm{p}=0.082)$.

In Group 1, $12 \%$ of seizures were intraoperative, $52 \%$ occurred within 0-7 days after surgery, and 36\% occurred within 8-30 days after surgery. The overall rate of intraoperative seizures in this study was $1.9 \%$. Among all patients, $11.5 \%$ had intraoperative cortical stimulation: $24 \%$ of Group 1 and 9.16\% of Group 2 ( $\mathrm{p}=0.044)$.

\section{Characteristics Associated With Perioperative Seizures}

We compared several patient characteristics between Groups 1 and 2 to identify any variables that were associated with perioperative seizures. Histological subtype (infiltrating glioma vs meningioma vs other; $p=0.04$ ), intradural tumor location $(\mathrm{p}<0.001)$, intraoperative cortical stimulation $(\mathrm{p}=0.004)$, and extent of resection $(\mathrm{p}=$ 0.002 ) were associated with the occurrence of perioperative seizures. Patients with infiltrating gliomas, intradural tumors, and STR or biopsy had higher odds of experiencing a perioperative seizure than patients with meningioma, extradural tumors, and GTR, respectively (Table 2). In addition, patients who underwent intraoperative cortical stimulation were more likely to have a perioperative seizure $(\mathrm{p}=0.004)$.

There was no significant association between the incidence of perioperative seizures and cerebral tumor location (Table 1). In addition, tumor laterality did not result in any significant difference between the 2 groups. More patients in Group 1 had an intradural tumor (84\%) compared with Group 2 (41.7\%, p = 0.0001). Within Group 1, intraoperative cortical stimulation was associated with timing of the seizure $(p=0.013)$. Of the 6 patients who received intraoperative cortical stimulation, 3 (50\%) experienced an intraoperative seizure, $2(33.3 \%)$ had a seizure within 0-7 days following surgery, and 1 (16.7\%) experienced a seizure within 8-30 days following surgery $(\mathrm{p}=0.013)$.

\section{Discussion}

The findings from this study suggest that there may be a subset of seizure-naïve brain tumor patients who ben- 
efit from prophylactic AEDs given their elevated risk of perioperative seizures. However, most seizure-naïve brain tumor patients do not appear to benefit from prophylaxis since patients with new-onset perioperative seizures made up only $4.6 \%$ of all seizure-naïve brain tumor patients treated at our institution. This may explain why no prior study has shown clear benefit to seizure prophylaxis in seizure-naïve brain tumor patients: the event rate was low, and studies were underpowered for such a low event rate. ${ }^{1,10,13,17,30,37,41}$

\section{Tumor Location}

Patients with intradural tumors had a higher risk of perioperative seizure. In contrast, extradural tumors were associated with freedom from perioperative seizures. Somewhat surprisingly, lobar involvement of the tumor did not show any association with perioperative seizures. The association between tumor location and seizures is well supported in the literature, however. Lee et al. found that patients with temporal lobe tumors were more than twice as likely to present with seizures.${ }^{19}$ However, that relationship was reflected only in slow-growing low-grade gliomas. Frontal, temporal, and parietal tumor locations are commonly associated with seizures; infratentorial and sellar tumors rarely cause seizures unless extension into the cerebral hemispheres is present. ${ }^{15}$ In a study investigating postoperative seizures in glioblastoma patients, Telfeian et al. concluded that the resection of frontal tumors and smaller tumors seemed to place patients at a higher risk for postoperative seizures. ${ }^{33}$ The lack of association between tumor cerebral lobe location and perioperative seizures in our study may be attributable to the selection of our patient population (seizure free prior to surgery, excluding posterior fossa and parasellar tumors) as well as the relatively small sample size.

\section{Histopathology and Extent of Resection}

There was an association between tumor histology and perioperative seizure incidence. Patients with infiltrating gliomas were at a higher risk for perioperative seizures than patients with meningioma or other primary CNS tumor. Moreover, all intradural tumors had a higher rate of seizure events than the extradural tumors. Tumor histology has been implicated in previous studies investigating perioperative seizures. Patients with indolent low-grade gliomas commonly present with seizures as the first clinical manifestation. ${ }^{27}$ A number of other factors have also been associated with a higher risk of seizures, including tumor type, tumor location, changes in the peritumoral environment, and various genetic factors ${ }^{36}$ However, the mechanisms underlying perioperative seizures in highgrade gliomas remain poorly understood. Patients can also develop seizures after surgery, including up to $20 \%$ and $23 \%$ of patients with meningioma and malignant glioma, respectively. ${ }^{4,21,38}$

Extent of resection in our study demonstrated findings similar to those previously reported in the literature. $5,6,8,9,25,27,40$ Patients with STR or brain biopsy were more likely to suffer a perioperative seizure than the patients with GTR.

\section{Cortical Stimulation}

We observed a significant association between intraoperative cortical stimulation and perioperative seizures. The incidence of intraoperative seizures associated with direct cortical stimulation has been reported to be $1.2 \%$ in brain tumor patients using the train-of-five technique and $9.5 \%$ with the $60-\mathrm{Hz}$ technique..$^{32}$ Furthermore, the incidence of stimulation-associated seizures is similar in brain tumor patients with symptomatic epilepsy and those without seizures. ${ }^{32}$ No risk factors for stimulation-associated seizures have been reported in the literature to date. Nonetheless, the overall rate of intraoperative seizures in our study was consistent with previously reported rates in the literature, with one average reported to be $9.5 \%$ (range $0 \%-24 \%)^{2,7,32}$ Patients with intraoperative seizures have been reported to have a longer hospital stay. ${ }^{29}$ However, intraoperative seizure incidence has not been associated with postoperative neurological deficits or an increase in seizure frequency. ${ }^{24,32}$ In contrast, seizures following tumor resection can have devastating consequences, often creating new neurological deficits or worsening existing deficits. $^{17}$

\section{Management Strategies}

The increased frequency of perioperative seizures in patients on phenytoin may reflect its complex, nonlinear pharmacokinetics. The half-life of phenytoin is estimated to be 14-31 hours, and approximately 1 week is required to achieve steady-state pharmacokinetics..$^{18}$ Thus, achieving a therapeutic plasma level is challenging, especially in acute presentations. ${ }^{3,11,24}$ The majority of Group 1 patients on phenytoin had subtherapeutic serum phenytoin levels, while a greater number of Group 2 patients on phenytoin had therapeutic levels. This difference trended toward a significant association with perioperative seizures and may explain why a greater number of Group 1 patients were on phenytoin. This suggests that seizure prophylaxis may be preventing seizures, at least in the setting of a patient on therapeutic levels of an AED.

This finding begs the question, what is the appropriate duration of perioperative seizure prophylaxis? Our findings suggest that the majority of new-onset seizures occur within the 1st postoperative week. This observation is congruent with the AAN recommendation to discontinue prophylactic AEDs in seizure-free patients after the 1st postoperative week, particularly in those who experience AED-related side effects. ${ }^{12}$

\section{Study Limitations}

A limitation of this retrospective study was the relatively small sample size of seizure events and, consequently, low statistical power reflected in marginally significant values. For this reason, a large prospective study of this patient population and factors associated with perioperative seizures is needed.

\section{Conclusions}

Perioperative seizures add significant morbidity to brain tumor patients, and seizure control is an important part of their management. Here, we investigated characteristics in 
seizure-naïve brain tumor patients who remained seizure free and those who had new-onset perioperative seizures. Infiltrating gliomas, supratentorial intradural tumors, and intraoperative cortical stimulation predisposed patients to perioperative seizures. Gross-total resection was an important factor in reducing the risk of perioperative seizures. While most seizure-naïve brain tumor patients do not benefit from perioperative seizure prophylaxis, such treatment should be considered in high-risk patients with supratentorial intradural tumors, in patients undergoing intraoperative cortical stimulation, and in patients in whom STR is likely.

\section{References}

1. Ansari SF, Bohnstedt BN, Perkins SM, Althouse SK, Miller JC: Efficacy of postoperative seizure prophylaxis in intraaxial brain tumor resections. J Neurooncol 118:117-122, 2014

2. Berger MS, Kincaid J, Ojemann GA, Lettich E: Brain mapping techniques to maximize resection, safety, and seizure control in children with brain tumors. Neurosurgery 25:786-792, 1989

3. Boarini DJ, Beck DW, VanGilder JC: Postoperative prophylactic anticonvulsant therapy in cerebral gliomas. Neurosurgery 16:290-292, 1985

4. Chaichana KL, Parker SL, Olivi A, Quiñones-Hinojosa A: Long-term seizure outcomes in adult patients undergoing primary resection of malignant brain astrocytomas. Clinical article. J Neurosurg 111:282-292, 2009

5. Chang EF, Potts MB, Keles GE, Lamborn KR, Chang SM, Barbaro NM, et al: Seizure characteristics and control following resection in 332 patients with low-grade gliomas. J Neurosurg 108:227-235, 2008

6. Choi JY, Chang JW, Park YG, Kim TS, Lee BI, Chung SS: A retrospective study of the clinical outcomes and significant variables in the surgical treatment of temporal lobe tumor associated with intractable seizures. Stereotact Funct Neurosurg 82:35-42, 2004

7. Conte V, Baratta P, Tomaselli P, Songa V, Magni L, Stocchetti N: Awake neurosurgery: an update. Minerva Anestesiol 74:289-292, 2008

8. Englot DJ, Berger MS, Barbaro NM, Chang EF: Factors associated with seizure freedom in the surgical resection of glioneuronal tumors. Epilepsia 53:51-57, 2012

9. Englot DJ, Han SJ, Berger MS, Barbaro NM, Chang EF: Extent of surgical resection predicts seizure freedom in lowgrade temporal lobe brain tumors. Neurosurgery 70:921928,2012

10. Forsyth PA, Weaver S, Fulton D, Brasher PM, Sutherland G, Stewart D, et al: Prophylactic anticonvulsants in patients with brain tumour. Can J Neurol Sci 30:106-112, 2003

11. Franceschetti S, Binelli S, Casazza M, Lodrini S, Panzica F, Pluchino F, et al: Influence of surgery and antiepileptic drugs on seizures symptomatic of cerebral tumours. Acta Neurochir (Wien) 103:47-51, 1990

12. Glantz MJ, Cole BF, Forsyth PA, Recht LD, Wen PY, Chamberlain MC, et al: Practice parameter: anticonvulsant prophylaxis in patients with newly diagnosed brain tumors. Report of the Quality Standards Subcommittee of the American Academy of Neurology. Neurology 54:1886-1893, 2000

13. Glantz MJ, Cole BF, Friedberg MH, Lathi E, Choy H, Furie $\mathrm{K}$, et al: A randomized, blinded, placebo-controlled trial of divalproex sodium prophylaxis in adults with newly diagnosed brain tumors. Neurology 46:985-991, 1996

14. Gokhale S, Khan SA, Agrawal A, Friedman AH, McDonagh DL: Levetiracetam seizure prophylaxis in craniotomy pa- tients at high risk for postoperative seizures. Asian J Neurosurg 8:169-173, 2013

15. Hauser WA, Annegers JF, Kurland LT: Incidence of epilepsy and unprovoked seizures in Rochester, Minnesota: 19351984. Epilepsia 34:453-468, 1993

16. Hildebrand J, Lecaille C, Perennes J, Delattre JY: Epileptic seizures during follow-up of patients treated for primary brain tumors. Neurology 65:212-215, 2005

17. Kong X, Guan J, Yang Y, Li Y, Ma W, Wang R: A metaanalysis: Do prophylactic antiepileptic drugs in patients with brain tumors decrease the incidence of seizures? Clin Neurol Neurosurg 134:98-103, 2015

18. Kuijlen JM, Teernstra OP, Kessels AG, Herpers MJ, Beuls EA: Effectiveness of antiepileptic prophylaxis used with supratentorial craniotomies: a meta-analysis. Seizure 5:291298, 1996

19. Lee JW, Wen PY, Hurwitz S, Black P, Kesari S, Drappatz J, et al: Morphological characteristics of brain tumors causing seizures. Arch Neurol 67:336-342, 2010

20. Leighton C, Fisher B, Bauman G, Depiero S, Stitt L, MacDonald D, et al: Supratentorial low-grade glioma in adults: an analysis of prognostic factors and timing of radiation. $\mathbf{J}$ Clin Oncol 15:1294-1301, 1997

21. Lieu AS, Howng SL: Intracranial meningiomas and epilepsy: incidence, prognosis and influencing factors. Epilepsy Res 38:45-52, 2000

22. Lote K, Stenwig AE, Skullerud K, Hirschberg H: Prevalence and prognostic significance of epilepsy in patients with gliomas. Eur J Cancer 34:98-102, 1998

23. Maschio M: Brain tumor-related epilepsy. Curr Neuropharmacol 10:124-133, 2012

24. Matthew E, Sherwin AL, Welner SA, Odusote K, Stratford JG: Seizures following intracranial surgery: incidence in the first post-operative week. Can J Neurol Sci 7:285-290, 1980

25. Meguins LC, Adry RA, Silva Júnior SC, Pereira CU, Oliveira JG, Morais DF, et al: Gross-total resection of temporal low grade gliomas is a critically important factor in achieving seizure-freedom. Arq Neuropsiquiatr 73:924-928, 2015

26. Moots PL, Maciunas RJ, Eisert DR, Parker RA, Laporte K, Abou-Khalil B: The course of seizure disorders in patients with malignant gliomas. Arch Neurol 52:717-724, 1995

27. Německová J, Němecek S, Náhlovský J, Parízek J, Sercl M: Epilepsy associated with low-grade brain glial neoplasms. Sb Ved Pr Lek Fak Karlovy Univerzity Hradci Kralove 38:175-183, 1995

28. North JB, Penhall RK, Hanieh A, Hann CS, Challen RG, Frewin DB: Postoperative epilepsy: a double-blind trial of phenytoin after craniotomy. Lancet 1:384-386, 1980

29. Nossek E, Matot I, Shahar T, Barzilai O, Rapoport Y, Gonen $\mathrm{T}$, et al: Intraoperative seizures during awake craniotomy: incidence and consequences: analysis of 477 patients. Neurosurgery 73:135-140, 2013

30. Shaw MD, Foy P, Chadwick D: The effectiveness of prophylactic anticonvulsants following neurosurgery. Acta Neurochir (Wien) 69:253-258, 1983

31. Sirven JI, Wingerchuk DM, Drazkowski JF, Lyons MK, Zimmerman RS: Seizure prophylaxis in patients with brain tumors: a meta-analysis. Mayo Clin Proc 79:1489-1494, 2004

32. Szelényi A, Joksimovic B, Seifert V: Intraoperative risk of seizures associated with transient direct cortical stimulation in patients with symptomatic epilepsy. J Clin Neurophysiol 24:39-43, 2007

33. Telfeian AE, Philips MF, Crino PB, Judy KD: Postoperative epilepsy in patients undergoing craniotomy for glioblastoma multiforme. J Exp Clin Cancer Res 20:5-10, 2001

34. Temkin NR, Dikmen SS, Wilensky AJ, Keihm J, Chabal S, Winn HR: A randomized, double-blind study of phenytoin 
for the prevention of post-traumatic seizures. N Engl J Med 323:497-502, 1990

35. Usery JB, Michael LM II, Sills AK, Finch CK: A prospective evaluation and literature review of levetiracetam use in patients with brain tumors and seizures. J Neurooncol 99:251-260, 2010

36. van Breemen MS, Wilms EB, Vecht CJ: Epilepsy in patients with brain tumours: epidemiology, mechanisms, and management. Lancet Neurol 6:421-430, 2007

37. Wu AS, Trinh VT, Suki D, Graham S, Forman A, Weinberg JS, et al: A prospective randomized trial of perioperative seizure prophylaxis in patients with intraparenchymal brain tumors. J Neurosurg 118:873-883, 2013

38. Yang P, Liang T, Zhang C, Cai J, Zhang W, Chen B, et al: Clinicopathological factors predictive of postoperative seizures in patients with gliomas. Seizure 35:93-99, 2016

39. Yuan Y, Peizhi Z, Maling G, Wu L, Yunhe M, Xiang W, et al: The efficacy of levetiracetam for patients with supratentorial brain tumors. J Clin Neurosci 22:1227-1231, 2015

40. Zaatreh MM, Firlik KS, Spencer DD, Spencer SS: Temporal lobe tumoral epilepsy: characteristics and predictors of surgical outcome. Neurology 61:636-641, 2003

41. Zachenhofer I, Donat M, Oberndorfer S, Roessler K: Periop- erative levetiracetam for prevention of seizures in supratentorial brain tumor surgery. J Neurooncol 101:101-106, 2011

\section{Disclosures}

The authors report no conflict of interest concerning the materials or methods used in this study or the findings specified in this paper.

\section{Author Contributions}

Conception and design: Ormond. Acquisition of data: Oushy. Analysis and interpretation of data: Ormond, Oushy, Sillau. Drafting the article: Oushy. Critically revising the article: Ormond, Oushy, Damek. Reviewed submitted version of manuscript: Ormond, Sillau, Ney, Damek, Youssef, Lillehei. Statistical analysis: Sillau. Administrative/technical/material support: Oushy. Study supervision: Oushy.

\section{Correspondence}

D. Ryan Ormond, Department of Neurosurgery, University of Colorado School of Medicine, 12631 E 17th Ave., Mail Stop C307, Aurora, CO 80045. email: david.ormond@ucdenver.edu. 